\title{
SOLUTION OF LINEAR ORDINARY DIFFERENTIAL EQUATIONS CONTAINING A PARAMETER ${ }^{1}$
}

\author{
ROBERT L. EVANS
}

Much work has been done in getting asymptotic solutions of certain linear ordinary differential equations containing a parameter. ${ }^{2}$ This note presents an important new application of the Cauchy-Kowalevsky theorem ${ }^{3}$ in the solution of such differential equations. In particular, a convergent solution is presented for the initial value problem in which

$$
y^{(n)}-\sum_{j=1}^{n} \lambda^{p i} P_{j}(\lambda, x) \cdot y^{(n-j)}=0
$$

and

$$
y^{(j)}(\lambda, 0)=(j !) \sum_{\alpha=0}^{\infty} b_{\alpha, j} \cdot \lambda^{-\alpha} \quad \text { for } j=0,1, \cdots, n-1
$$

where:

$$
P_{j}(\lambda, x)=\sum_{\mu=-p m_{j}}^{\infty} \sum_{\nu=[(p-\mu-1) / p]}^{\infty} a_{j, \mu, \nu} \lambda^{-\mu} x^{\nu}
$$

(ii) the given series in (2) and (3) converge wherever $|x|<x_{0}$ and $|\lambda|>\lambda_{0}$,

(iii) $n$ and $p$ are positive integers and the $m_{j}$ 's are non-negative integers, and

(iv) $x$ is a complex variable while $\lambda$ is a complex parameter.

In the present treatment (1) and (2) are considered in a certain neighborhood of $x=0$ and, for the parameter $\lambda$, in a neighborhood of $\lambda=\infty$. The point at $x=0$ is said to be a regular point of (1), and whenever one or more $m_{j}$ is positive, $x=0$ is also said to be a turning, point of (1).

The transformations

$$
z=\lambda^{p} x \text { and } y(\lambda, x)=y\left(\lambda, z / \lambda^{p}\right)=w(\lambda, z)=w\left(\lambda, \lambda^{p} x\right)
$$

Presented to the Society, April 26, 1952; received by the editors March 3, 1952 and, in revised form, May 2, 1952.

1 This work was supported by Army Ordnance contract DA11-022-ORD-489, for which the author is grateful. It is also an outgrowth of the author's thesis which was written under the helpful advisorship of Dr. H. L. Turrittin.

2 For the work of some of the more recent authors see the bibliography.

${ }^{3}$ S. v. Kowalevsky, Journal für Mathematik vol. 80 (1875) pp. 1-32. 
convert (1) to

$$
w^{(n)}=\sum_{j=1}^{n} P_{j}\left(\lambda, z / \lambda^{p}\right) w^{(n-j)}=0 .
$$

This transformation has been used only occasionally in the past and it is presented here in a way that shows how much it simplifies the treatment of otherwise difficult problems. This point is illustrated clearly but briefly in subsequent examples.

The solution of (4) (when (2) is also considered) is

$$
w(\lambda, z)=\sum_{\alpha=0}^{\infty} \sum_{\beta=0}^{\infty} b_{\alpha, \beta} \lambda-\alpha z^{\beta}
$$

if the $b$ 's not given in (2) are defined by solving for $b_{q, r+n}$ in the following equation when $q, r=0,1,2, \ldots$. Thus

$$
\begin{aligned}
(r+1) \cdot(r+2) \cdots(r+n) b_{q, r+n} & \cdots(r-\nu+n-i) ! \\
& =\sum_{j=1}^{n} \sum_{\mu=-p m_{j}, \nu} \sum_{\nu-[(p-\mu-1) / p]}^{r} \frac{(r-p)}{(r-\nu) !} b_{q+p \nu-\mu, r-\nu+n-j} \\
& -\sum_{j=1}^{n} \sum_{\mu=\mu_{0}}^{q} \sum_{\nu=[(q+p-\mu) / p]}^{r} \frac{(r-\nu+n-i) !}{(r-\nu) !} a_{j, \mu, \nu} b_{q+p \nu-\mu, r-\nu+n-j}
\end{aligned}
$$

if $\mu_{0}=\max \left\{-p m_{j},(q-p r)\right\}$,

$$
[(p-\mu-1) / p]=\left\{\begin{array}{l}
\text { greatest integer contained in } \\
((p-\mu-1) / p), \mu<p-1 \\
0, \mu \geqq p-1,
\end{array}\right\},
$$

and if $[(q+p)-\mu) / p]$ is defined similarly.

The region of convergence for the solution given by (5), together with (2) and (6), is easily extended beyond the range given for this case by the original Cauchy-Kowalevsky theorem. ${ }^{3}$ Successive application to $z$ (with $\lambda$ fixed) and to $\lambda$ (with $z$ fixed) of a well known method of treatment $t^{4}$ yields the result that the series in (5) is convergent for all finite $z$ and for all $\lambda$ such that $|\lambda|>\lambda_{0}$.

In terms of the original problem, (1) and (2), the final solution is

$$
y(\lambda, x)=\sum_{\alpha=0}^{\infty} \sum_{\beta=0}^{\infty} b_{\alpha, \beta} \lambda^{p \beta-\alpha} x^{\beta}
$$

which converges wherever $|x|<x_{0}$ and $|\lambda|>\lambda_{0}$. In using this result

${ }^{4}$ E. T. Copson, Theory of functions of a complex variable, Oxford University Press, 1935, pp. 85-86. 
it is helpful to note that for many subcases of (1) the parts of (7) resulting when $\alpha$ is a fixed single value, namely $\sum_{\beta=0}^{\infty} b_{\alpha, \beta}\left(\lambda^{p} x\right)^{\beta}$, are forms of well known and tabulated functions.

For example, when, in (1), $n=2, p=m_{2}=1, P_{1}(\lambda, x) \equiv 0$, and $a_{2, \mu, \nu}=0$ for all $\nu$ whenever $\mu=0$ or $1(\bmod 3)$, then T. M. Cherry's results [3] show the relation of (7) to Bessel and Airy functions. The present results give a simple expression of those that Cherry obtained when his $z$ variable is bounded-because our $x$ is the analogue of that variable.

In independent and almost simultaneous work, R. E. Langer [2] studied the generalization of Cherry's case that arises when, in (1), $n=p=2, m_{2}=1, P_{1}(\lambda, x) \equiv 0$, and $a_{2, \mu, \nu}=0$ for all $\nu$ whenever $\mu=0$ or $2(\bmod 3)$. In Langer's work the Bessel function representations were used. Since Langer's $\xi^{2}$ is most closely related to the $z$ of the present treatment, the result in (7) relates to those that Langer found for bounded values of his $\xi$ (his $|\xi| \leqq N$ ).

The author generalized Langer's case [5] in the form of that subcase of (1) in which $n=p=2, m_{2}=1$, and $P_{1}(\lambda, x) \equiv 0$. The results of that study are related to ( 7$)$ in the same way as Langer's and Cherry's. That is, for all three cases the present results confirm and can replace the earlier ones corresponding to bounded values of our $z$ (or $\lambda^{p} x$ ). However, the present results would need extension before they could be related to the earlier asymptotic solutions pertaining to unbounded values of our $z(|z|>N)$.

\section{BibliogRAPHY}

1. W. J. Trjitzinsky, Acta Math. vol. 67 (1936) pp. 1-50.

2. R. E. Langer, Trans. Amer. Math. Soc. vol. 67 (1949) pp. 461-490.

3. T. M. Cherry, Trans. Amer. Math. Soc. vol. 68 (1950) pp. 224-257.

4. W. Wasow, Ann. of Math. vol. 52 (1950) pp. 350-361.

5. R. L. Evans, Bull. Amer. Math. Soc. Abstract 56-1-48 and University of Minnesota thesis, April 1951.

UNIVERSITY OF MiNNESOTA 